\title{
Within-session dynamics of categorical and memory mechanisms in pigeons
}

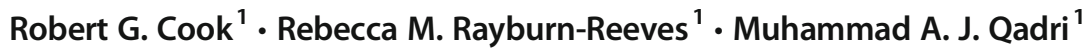

Accepted: 28 October 2020 / Published online: 2 December 2020

(C) The Psychonomic Society, Inc. 2020

\begin{abstract}
The current experiments used categorical mid-session reversal (MSR) to examine how eight pigeons utilized categorical and item-specific mechanisms to learn and solve a novel variation of this task. Employing a fixed order of trial-unique pictorial items from two categories (flowers and cars) on each simultaneous discrimination trial, categorical and item-specific information was available during each session's 80 trials. Choices to one category were rewarded for the first 40 trials, after which the correct category was reversed (e.g., car correct early $\rightarrow$ flower correct late). This procedure selectively impacts the time-modulated utility of categorical identification, but leaves exclusively item-specific information intact. Results revealed that categorical control emerged rapidly and before item-specific memorization, which came after extended experience. Both types of control occurred within a session, with control modulated by their time-based relative utility. The implications for the timing, ordering, and attention by animals to categorical and item-specific information is considered.
\end{abstract}

Keywords Categorization $\cdot$ Memorization $\cdot$ Cognitive flexibility $\cdot$ Pigeons

\section{Introduction}

Categorization and memorization are critical cognitive capacities for any complex organism. Categories permit the rapid organization of the world into meaningful units and adaptive responding to new circumstances using their similarity to prior experiences. In contrast, memorizing instances allows for precise responding to specific information and can partially support categorization behavior. Many studies have found separate support for these capacities across animals (e.g., Cook, Levison, Gillett, \& Blaisdell, 2005; Fagot \& Cook, 2006; George \& Pearce, 2012; Herrnstein \& Loveland, 1964; Wasserman, Kiedinger, \& Bhatt, 1988). These processes are not mutually exclusive, however, and organized behavior is likely formed by their joint function. Consequently, we suggest a shift towards identifying the conditions that dictate when and which of these processes are employed and how they work in coordination or opposition.

Beyond the extensive studies of humans, pigeons have been the primary animal model for examining these general

Robert G. Cook

Robert.Cook@tufts.edu

1 Department of Psychology, Tufts University, 490 Boston Ave., Medford, MA 02155, USA questions. Using a category/exception task, Cook and Smith (2006) found that pigeons initially abstracted the common properties of categorical stimuli before learning to memorize the individual exemplars (i.e., the exception). This ordering of category-level to exemplar-specific processing has been similarly found in humans (Minda \& Smith, 2000; Smith \& Minda, 1998), suggesting a fundamental similarity in the sequential organization of these processes across species. In contrast, Katz and Wright (2006) explored how the number of training exemplars influenced same/different discrimination and categorization in pigeons. They found using fewer exemplars in training produced item memorization, while increasing training set sizes yielded better transfer performance to novel items consistent with the use of a rule-based category. Thus, whether pigeons use summary or item-specific representations of their experience may depend on factors that include category structure, category size, the nature of the discrimination task, and the degree of (over)learning.

Our experiments report here new evidence regarding pigeons' dynamic use of summary-based and instance-specific feature information in solving a categorical mid-session reversal (MSR) task. MSR is a recently studied phenomenon in which a learned discrimination is reliably reversed midway through a session (Cook \& Rosen, 2010). Instead of using trial outcomes (e.g., win-stay/lose-shift), pigeons consistently utilize internal timing mechanisms, despite extensive training, to switch between the 
competing representations needed to correctly respond to the contrasting segments of a session (Rayburn-Reeves \& Cook, 2016). This temporal solution yields both anticipatory and perseverative errors around the reversal boundary, resulting in a characteristic psychophysical function marking the competitive transition from the control of the solution for the first segment of a session to that employed for the second.

We used a novel variation of this procedure involving the discrimination of two complex pictorial categories, rather than simple visual or spatial stimuli. This categorical variation allowed us to diagnose the shifting nature of the controlling features used to group and discriminate items within each category and their use across a session. We trained eight pigeons to discriminate between car and flower categories in an MSR procedure using a fixed order of the pictorial stimuli. Each trial simultaneously presented one flower and one car picture. In the first half of each session, a choice peck to one category was rewarded (C1) and in the second half, choice of the other category $(\mathrm{C} 2)$ was rewarded. Critically, the pictures for each trial were tested in the same order each session (see Fig. 1).

This combination of categorical stimuli and fixed sequential arrangement allows both category-level and item-specific information to be continuously useful to the animal. If the pigeons attend to category-level features, systematic anticipation and preservation errors should be produced by competitive interference tied to the reversal of the categoryreinforcement relationship at the session's midpoint. Alternatively, attention to item-specific features should permit highly accurate performance throughout the session without exhibiting any impact on accuracy from the overarching categorical reversal. Therefore, the pattern of responding before, during, and after the reversal boundary, both within and across sessions, should reveal how the birds attend to and use category-level features to group similar items and itemspecific features to distinguish among them during their acquisition and performance of this MSR discrimination.

We report that categorical learning emerges rapidly to control initial choice behavior, followed by item-specific learning with further experience. Additionally, different diagnostic tests revealed that the control by these different feature sets varied dynamically within a session depending on its momentary utility as caused by the categorical reversal at the midpoint.

\section{Method}

\section{Animals}

Eight male White Carneaux pigeons were tested (maintained at $80-85 \%$ of their free-feeding weight). Four were naïve and four had experience with a spatial, mid-session reversal task

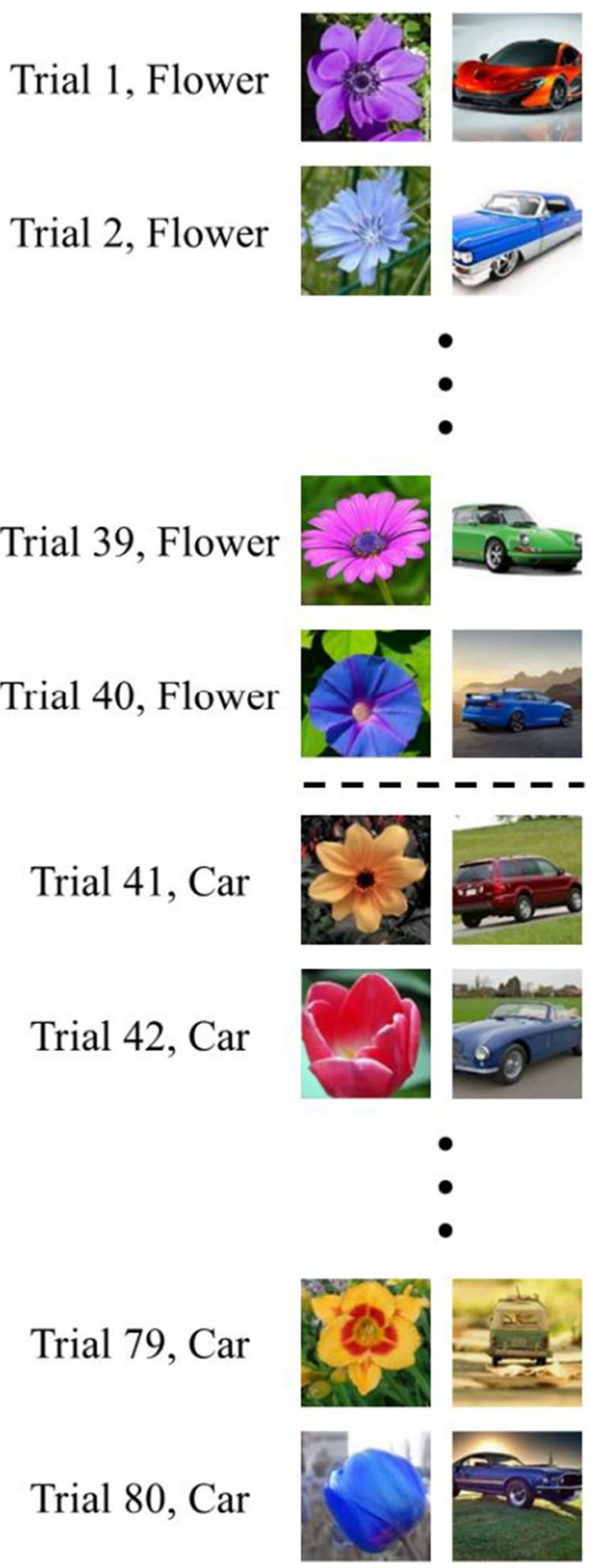

Fig. 1 Depiction of a categorical mid-session reversal task with a trialspecific picture sequencing. Each row indicates the trial number, an example correct response with "flower" as the first correct category $(\mathrm{C} 1)$, and a trial-specific combination of pictures from each category (e.g., Category 1 Stimulus $1-\mathrm{C} 1 \mathrm{~S} 1$ vs. Category 2 Stimulus $1-\mathrm{C} 2 \mathrm{~S} 1$; $\mathrm{C} 1 \mathrm{~S} 2$ vs. C2S2; C1S3 vs. C2S3). The dashed line illustrates the location of the reversal boundary between the two segments of a session, though no such indicator or signal is present in the experiment. All eight pigeons received the same 80 images, but each pigeon was assigned one of four sequences. In the experiment, car and flower positions were right/left counterbalanced within a segment

using colored intertrial-interval (ITI) cues (Rayburn-Reeves, Qadri, Brooks, Keller, \& Cook, 2017). This sample size is comparable to the size of groups used in other mid-session 
reversal experiments (see review in Rayburn-Reeves \& Cook, 2016). All methods were approved by the IACUC at Tufts University.

\section{Apparatus}

Training and testing were conducted in a computer-controlled (Dell Optiplex GX260) PVC test chamber measuring $40 \times 40$ $\times 40 \mathrm{~cm}$. The front panel of the chamber had an infrared touchscreen (ezscreen; Houston, Texas) for recording pecks towards the lower $34 \times 25 \mathrm{~cm}$ portion of the color LCD monitor situated behind it (Dell 1908FPt, 1,280 × 1,024 resolution). A $28 \mathrm{~V}$ house light located in the ceiling was illuminated, except during timeouts. Food was accessible below the touchscreen, controlled by a food hopper (Coulbourn Instruments) containing mixed-grain reward.

\section{Procedure}

Initial training After the four naïve pigeons were autoshaped to peck the ready stimulus, all eight pigeons were initially given two 80-trial shaping sessions where only the correct stimulus appeared on each trial. Trials started with a white circular ready stimulus $(2.7 \mathrm{~cm}$ diameter $)$ appearing in the center of the screen. After a single peck or $60 \mathrm{~s}$, it was turned off and a $5.7 \times 5.7 \mathrm{~cm}$ picture appeared randomly on the right or left side of the screen. A single peck at the picture resulted in immediate reward of 3-s access to mixed grain. If there was no response after $20 \mathrm{~s}$, the picture was removed and a reward was automatically delivered. All birds quickly learned to peck the pictures and once shaping was completed MSR training began.

Categorical MSR training Each MSR session consisted of 80 trials. Each trial began with a ready signal. After a single peck, it was replaced by two pictures, one of a flower and one of a car, vertically centered and equidistant from the center of the screen, separated by $5.7 \mathrm{~cm}$. A single peck to either picture was considered a choice and turned off both pictures. During the first 40-trial segment a choice peck to pictures from each bird's correct first category $(\mathrm{C} 1)$ resulted in grain reward (flowers for pigeons \#1B, \#2B, \#3L \& \#4S; cars for \#5C, \#6G, \#7M \& \#8W). A choice peck to a picture from the other category $(\mathrm{C} 2)$ was incorrect and resulted in a dark 5-s time out. For trials 41 to 80 (i.e., the second segment), these categorical choice contingencies were reversed for each bird (i.e., categorical MSR). Trials were separated by a 5 -s ITI.

Each of the 80 trials comprising a daily session displayed a trial-specific car and flower picture (see Fig. 1; i.e., Trial 1 $\mathrm{C} 1 \mathrm{~S} 1$ vs. C2S1; Trial $2-\mathrm{C} 1 \mathrm{~S} 2$ vs. C2S2). A total of $80 \mathrm{car}$ and 80 flower pictures were used. Four different orderings of the stimuli was used (one per two birds, each with differing $\mathrm{C} 1$ assignments). Thus, for given a bird, the pictures were in the same ordinal trial position each session, although their right/ left position was randomized across sessions constrained such that each category appeared on either side an equal number of times during each session half. This baseline organization of fixed-position categorical MSR training was conducted for 140 completed baseline sessions (for two birds, ratio strain and apparatus errors caused incomplete sessions, which were discarded). The exchange tests described next were interspersed within these baseline sessions.

Trial exchange tests In three sets of tests, trials were repositioned to determine how the birds responded to the same exact flower and car pair when they appeared at different times (i.e., different trials) in a session. During these transposition tests, selected trials were swapped in their ordinal positions within the standard 80-trial session. For example, the stimuli normally presented on Trial 2 were instead presented at Trial 79 , and in the same session stimuli normally presented on Trial 79 were presented on Trial 2 (i.e., Trials 2 and 79 were "exchanged"). By the end of testing, trials normally encountered at the beginning, middle, or end of each session had been repositioned to each of these other points within and across the reversal boundary (see Online Supplementary Material for precise trial numbers tested and order of tests). During exchange trials, no choices were reinforced. This exchange testing started after the first novel transfer test described in the next section.

Novel-item transfer tests Three test sets presenting novel items assessed control by category membership (i.e., categorical transfer) at different points during training. The first set tested novel items in the middle of each categorical segment to assess overall categorical control early in training, after a bird had completed 20-35 baseline sessions. The second test placed novel items near both endpoints of the categorical segments (e.g., trials 2, 39, 41, and 79; see Online Supplementary Material for precise trial numbers tested) to evaluate the nature of the mechanism employed at the moments of least and most time-based ambiguity. This second test set was conducted later in training, on average 77 sessions after the first novel test. The third test set used sessions of entirely novel items to measure the degree of categorical control after the emergence of item-specific responding and was tested at the end of training. All novel trials were non-differentially reinforced, with any and all choices rewarded. Sessions were otherwise conducted using the same procedures as previously described.

\section{Results}

Categorical MSR training Learning of the categorical MSR was rapid. Figure 2 shows acquisition indexed by the mean percentage of $\mathrm{C} 1$ choices. The left panel shows mean $\mathrm{C} 1$ 


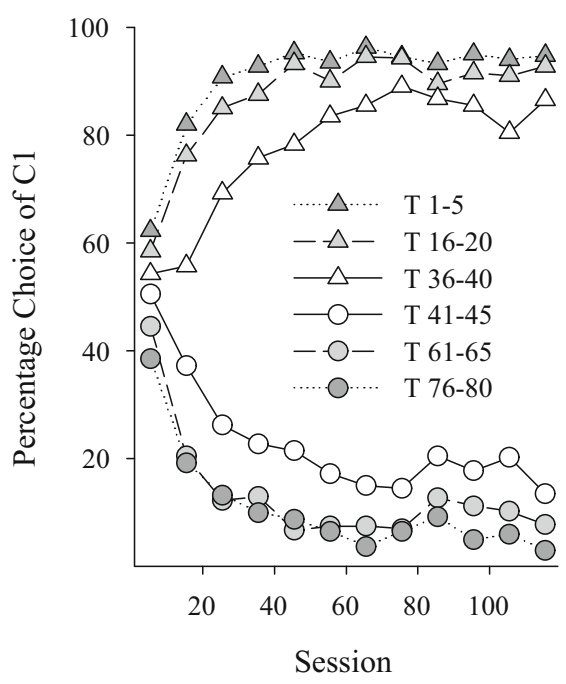

Fig. 2 Mean percentage of $\mathrm{C} 1$ choices as functions of time within and across sessions. The left panel shows the mean percentage of $\mathrm{C} 1$ choices over training sessions for select five-trial blocks within a session. The right panel shows the mean percentage of $\mathrm{C} 1$ choices of all five-trial

responding over sessions. The marked divergence of $\mathrm{C} 1$ responding across the two segments evidences discrimination learning and its reversal. Depicting the session-wide choice psychophysics, the right panel shows mean $\mathrm{C} 1$ responding within sessions across training.

Birds first learned the endpoints of the discrimination. This learning is evident in the change in mean $\mathrm{C} 1$ responding in the first 20 trials of the first ten-session block (63.1\%) compared to the last 20 trials $(37.0 \%), t(7)=5.3, p=.001, d=1.9$. The proportion of $\mathrm{C} 1$ responses in the first ten sessions decreased within the session (right panel; significant downward slope to the best-fitting line $t(7)=4.9, p=.002, d=1.7)$. The increasing presence of anticipatory and perseverative errors near the reversal boundary provides clear evidence that category identity controlled their behavior early in training.

Extended training improved accuracy, especially near the reversal boundary (Fig. 2, right). Over sessions 131-140, for example, the birds almost immediately reversed their choice behavior at the reversal boundary (mean $\mathrm{C} 1$ responses for the ten trials before the reversal $90.4 \%$, vs. $9.5 \%$ after the reversal). Consistent with this, a repeated-measures ANOVA analyzing $\mathrm{C} 1$ responding over five-trial bins and across ten-session blocks identified a significant Trial $\times$ Session interaction, $F(195,1365)$ $=18.1, p<.001, \eta_{\mathrm{p}}^{2}=.721$. This reduced categorical interference at the boundary suggests that specific stimulus identity was likely supporting choice behavior. Together these training data suggest that categorical information controlled early choice behavior while the learning of item-specific information emerged much later to enhance accuracy.

Trial exchange testing Table 1 displays mean $\mathrm{C} 1$ choice behavior for the exchange tests as a function of their original

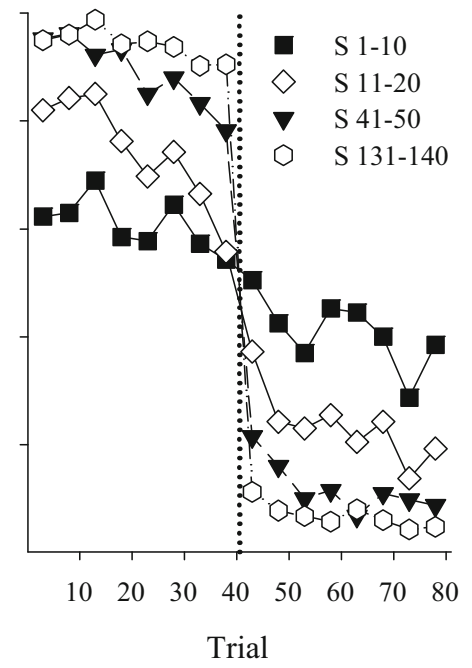

blocks within a session for select ten-session blocks across training. The location of the reversal boundary in the middle of the session is indicated by the dotted line in the right panel

position and exchanged destination. The last column infers the type of category response made at the destination by whether responding was numerically greater $(\mathrm{C} 1)$ or less (C2) than 50\%. These results converge with a hypothesis that control by categorical information dominates at the beginning and end of a session, but item-specific information dominates near the mid-session reversal boundary.

Most revealing of this pattern are the trials moved from the beginning (B) to the end (E) of the session (B-E) and vice versa (E-B). In these cases, the item-specific reward histories should cause the pigeons to make choices appropriate to the origin of the exchanged item, while its category status would cause them to respond consistently with its destination. Responding showed significant categorical control by the destination, despite the specific history of punishment these responses received at the original training locations. Early $\mathrm{C} 1$ items showed strong $\mathrm{C} 2$ control when tested at the end and vice versa for late $\mathrm{C} 2$ trials tested at the beginning (see Table 1), and both were significantly different from their history of rewarded behaviors, $t \mathrm{~s}(7)>12.7, p \mathrm{~s}<.001, d \mathrm{~s}>4.4$. A companion effect was found with items from the beginning and end of the session in supporting poorer accuracy when swapped with near-boundary items in the same segment (B$\mathrm{MC} 1, \mathrm{E}-\mathrm{MC} 2, t \mathrm{~s}(7)>2.7, p \mathrm{~s}<.031, d \mathrm{~s}>0.9)$. This latter effect further suggests the pigeons processed the endpoint items differently such that item-specific information was not sufficiently processed or memorized to support high accuracy near the reversal.

In contrast, when trials from the middle of a session were swapped across the reversal boundary (MC1-MC2, MC2$\mathrm{MC1}$ ), the pigeons' behavior was consistent with item memorization. While not as accurate as when tested at their origin, 
Table 1 Results of trial exchange tests

\begin{tabular}{|c|c|c|c|c|c|}
\hline Condition & Origin & $\begin{array}{l}\text { Origin } \\
\text { C1 choice } \%\end{array}$ & New location & $\begin{array}{l}\text { New location } \\
\mathrm{C} 1 \text { choice } \%\end{array}$ & Test classification \\
\hline \multicolumn{6}{|c|}{ Beginning and end positions } \\
\hline $\mathrm{B}-\mathrm{MC} 1$ & C1-Begin & 94.1 & C1-Mid & 58.6 & $\mathrm{C} 1$ \\
\hline B-MC2 & C1-Begin & 92.2 & C2-Mid & 53.1 & $\mathrm{C} 1$ \\
\hline B-E & C1-Begin & 94.5 & C2-End & 20.3 & $\mathrm{C} 2$ \\
\hline E-MC2 & C2-End & 11.3 & C2-Mid & 28.1 & $\mathrm{C} 2$ \\
\hline E-MC1 & C2-End & 7.8 & C1-Mid & 26.6 & $\mathrm{C} 2$ \\
\hline E-B & C2-End & 8.6 & C1-Begin & 90.6 & $\mathrm{C} 1$ \\
\hline \multicolumn{6}{|c|}{ Middle positions } \\
\hline MC1-B & C1-Mid & 67.6 & C1-Begin & 94.5 & $\mathrm{C} 1$ \\
\hline MC1-MC2 & C1-Mid & 82.0 & C2-Mid & 70.3 & $\mathrm{C} 1$ \\
\hline MC1-E & C1-Mid & 81.3 & C2-End & 35.9 & $\mathrm{C} 2$ \\
\hline MC2-E & C2-Mid & 23.0 & C2-E & 14.8 & $\mathrm{C} 2$ \\
\hline MC2-MC1 & C2-Mid & 21.1 & C1-Mid & 31.3 & $\mathrm{C} 2$ \\
\hline MC2-B & C2-Mid & 15.6 & C1-Begin & 82.8 & $\mathrm{C} 1$ \\
\hline
\end{tabular}

C1 first segment correct category, $C 2$ second segment correct category, B/Beginning trial from or placed in early portion of session, $M C 1 / M C 2 / M i d$ trial from or placed in middle portion of session, specifically in the C1-correct or C2-correct half, E/End trial from or placed at end of session - e.g., B-MC1 indicates a trial originating from the beginning and tested at the reversal within the first segment

See Online Supplemental Table 1 for confidence intervals

$t(7)=2.7, p=.029, d=1.0$ and $t(7)=2.6, p=0.035, d=0.9$, the overall classification of these trials remained consistent with their original training $(82.0 \%$ to $70.3 \% ; 21.1 \%$ to $31.3 \%)$. This contrasts with the endpoint exchanges, where the items were completely reclassified. Correspondingly, when these middle items were moved to their matching endpoints (MC1-B, MC2-E), their classification was significantly enhanced, $t \mathrm{~s}(7)>2.5, p \mathrm{~s}<.039, d \mathrm{~s}>0.9$, and when relocated to the other segment's endpoint (MC1-E; M2-B), their classification was reversed from their training experience, $t \mathrm{~s}(7)>7.9, p \mathrm{~s}<.001, d \mathrm{~s}>2.8$. Like the prior learning results, these exchange tests suggest categorical control was strongest at the session endpoints, while item-specific information was more influential near the reversal boundary.

Novel transfer testing These novel-item transfer tests provide further evidence for this same shifting pattern of control between types of information by evaluating the pigeons' use of categorical information throughout their training (see Fig. 3). The first test phase occurred early in training (sessions 31-40) and captured the early development of control by category features as suggested by the choices made during acquisition. In this first test, mean $\mathrm{C} 1$ responding to the novel items in first $(61.7 \%)$ and second $(39.8 \%)$ segments differed significantly, $t(7)=2.5 ; p=.041, d=0.9$, though not as much as familiar baseline items. Thus, the pigeons generalized to novel stimuli utilizing category membership early in training, although they did detect the novelty.
After 100 further training sessions, novel testing was repeated. Having established differential control across a session during exchange testing, novel items were now inserted at the endpoints and middle of the session to evaluate the nature of the control at these time points. The novel items at the two endpoints exhibited strong category-consistent responding with mean $\mathrm{C} 1$ responding (first segment $-78.1 \%$, second segment $-21.9 \%$ ) being significantly different from each

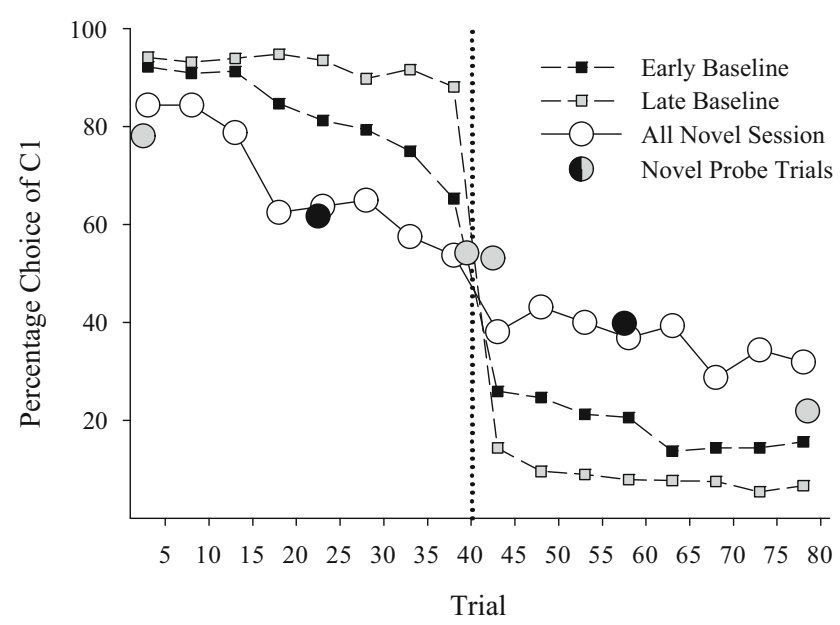

Fig. 3 The mean percentage of $\mathrm{C} 1$ choices over three different tests with novel items. Black symbols depict results from tests early in training. Gray symbols depict results from tests late in training. The curve depicted with white symbols comes from sessions in which all items were novel. The reversal boundary is indicated by the dotted line 
other, $t(7)=6.0, p<.001, d=2.1$, and from $50 \%$ (first segment, $t(7)=5.4, p=.001, d=1.9$; second segment, $t(7)=4.7$, $p=.002, d=1.7)$. In contrast, mean $\mathrm{C} 1$ responding to the novel items placed near the reversal $(\mathrm{M}-\mathrm{C} 1=54.2 \%$ and $\mathrm{M}-\mathrm{C} 2=53.1 \%$ ) were not significantly different from $50 \%$ or each other $(t \mathrm{~s}(7)<.7, \mathrm{ps}>.516)$, consistent with itemspecific control.

The third test examined choice behavior with sessions composed of exclusively novel items, removing any history of item-specific learning. Figure 3 shows the pigeons correctly classified novel items at the beginning and end of a session, but did so more poorly in the middle. This latter performance can be contrasted to the strongly demarcated control exhibited for the comparable (late) baseline items. Novel items at the two endpoints exhibited strong categorical control with mean $\mathrm{C} 1$ responding (start first segment $\mathrm{M}=84.3 \%$ and end second segment $\mathrm{M}=33.1 \%$ ) being significantly different from each other $t(7)=14.0, p<.001, d=5.0$, from $50 \%$ (first segment $t(7)=17, p<.001, d=6.1$; second segment $-t(7)=4.4, p=$ $.003, d=1.6$ ), and significantly different from baseline (first segment $-t(7)=3.7, p=.007, d=1.3$; second segment $-t(7)=$ $7.9, p<.001, d=2.8)$.

\section{General discussion}

This experiment revealed four new results regarding how pigeons deploy attention to categorical and item-specific features in a complex categorical MSR discrimination, which have important implications for the structure of their learning mechanisms. First, categorical-level control emerged rapidly at the onset of training, with similar items being rapidly grouped by a set of common features. Second, control by item-specific information emerged later with experience, as items near the reversal boundary were increasingly discriminated near-perfectly. Third, by the end of training, both types of feature control were simultaneously present within different portions of the same session. Fourth, the deployment of attention to these two types of features depended on their relative utility at different points within a session. Categorical-level control was best exhibited at the session endpoints, where its shared common features were most efficient, while control by item-specific features was strongest during the mid-session trials near the reversal, where categorical features would result in the greatest interference and itemlevel information allowed for the best discrimination among the stimuli.

The shape of the psychophysical choice functions and the distribution of errors near the reversal provide the strongest evidence that categorical representations of common features controlled the pigeons' choices early in training. The interference seen at the reversal boundary is typical of MSR experiments using simple colors (Rayburn-Reeves \& Cook, 2016) or complex rules (Daniel, Cook, \& Katz, 2015). The initial random assignment of the stimuli across birds to their trial positions precludes the unlikely possibility that the items at the reversal point just happened to be harder or more in need of memorization than those at the session endpoints. Instead, the faster learning at both endpoints and the competitive interference at the reversal suggest early on that attention to the shared characteristics within the two categories was most critical. Diagnostic testing with novel items and transposed trials further corroborated this categorical account.

With additional experience the pigeons produced fewer errors, with a considerable reduction in categorical interference at the reversal boundary. The pigeons' memorization of the item-specific features of the stimuli situated near the reversal boundary best accounts for this improvement, since such attention to detail could overcome the interference caused by the competing categorical features around the reversal boundary. This account is also supported by the poor performance with familiar or untrained category-consistent items near the boundary and the persistent tracking of the original trained identity when items were exchanged near the reversal boundary. This sequential acquisition of categorical-like representations followed by item-specific ones mirrors the results of Cook and Smith (2006) and echoes the relational versus itemspecific time course of Gibson and Wasserman (2004).

While both sets of features were simultaneously present across a session, their use was modulated across a session. Categorical features exerted strong control at the endpoints of sessions, as clearly evidenced in the novel transfer and exchange tests. Item-specific features strongly exerted control near the reversal. Critically, a purely item-specific account of these results or a purely categorization account would fail to explain this pattern of results, necessitating a within-session attentional shift between these underlying feature spaces. Rayburn-Reeves et al. (2017) found a similar dynamic within-session control of choice by informative colored context cues presented during the ITI. In their study, the reliance on internal time-based cues shifted to the disambiguating contextual ITI cues near the reversal boundary as revealed by a series of miscuing trials. We assume that our differential control across the session was primarily mediated by internal timing cues as typically found in MSR experiments with pigeons, although item-specific information could have contributed here, too. An asymmetry between early and late items tested near the reversal boundary interior suggests that some item-specific processing may have occurred toward the end of the session, consistent with noisy time perception mediating featural attention. More 
important, however, is that this dynamic modulation of control followed a trajectory correlated with, or possibly determined by, the relative choice utility of each set of features (cf. Cook, Brown, \& Riley, 1985). The pigeons switched between these modes of control, using memorized individuating information when it served to resolve the conflicting information presented by shared category membership at the reversal and using categorical information when it best served to classify the larger number of items at the end points.

Overall, our results clearly suggest that two different encoding feature spaces are present. One feature space mediates the shared membership identification of the categories, and the other facilitates the individuating itemspecific discrimination. Their separate use seems demanded by the diverging utility of these feature sets within the same stimuli. The features most diagnostic in determining category membership are unlikely to provide the best leverage for item-specific recognition at the reversal boundary. Successful elimination of interference at the boundary requires the birds to suppress attention to unifying categorical features and instead attend to differentiating item-specific features that are likely categoryirrelevant. Nosofsky (1987), among others, suggested that attention can change or stretch a feature space to facilitate category-like behavior. A similar change in attentional weights to different features in non-overlapping spaces seems required to resolve the divergent and conflicting demands of simultaneously using category membership and specific identity at different points within this form of MSR task.

These findings make contact with several related sets of hypotheses. The first is the relationship between prototype and exemplar-based notions of categorization that have been proposed to explain how human and nonhuman animals learn categorical discriminations (Ashby, 1992). One class of solutions consists of abstraction-like mechanisms that condense categorical experiences into a form of summary representation that mediates recognition of new members. The second class consists of separate stored exemplar-based representations that are used collectively to generalize behavior to new items. Our findings suggest that perhaps these accounts may co-exist and co-function, with summary, possibly prototype-like, representations based on shared features emerging quickly and stronger exemplar encoding occurring later. We have attempted not to confuse the instance learning required here with the type of exemplar learning that is proposed to support categorization. Computational models with distinct summary mechanisms and instance-based learning can likely succeed in producing this pattern of data, for example by incorporating time as a contextual feature used to modulate behavior. That said, both mechanisms likely originate in associating features with specific outcomes in long-term memory (Cook et al., 2005).

These results also contact research on rule-based learning and classification. Ashby and his colleagues have suggested humans have two distinct category mechanisms: one that uses slower associatively based implicit connections of items to outcomes, and an evolutionarily newer, second system that can quickly and explicitly generate rules. In collaboration, we used tasks that identify both mechanisms in humans to reveal that pigeons exhibit only one associatively based mechanism (Qadri, Ashby, Smith, \& Cook, 2019; Smith et al., 2011). This singular classification mechanism, however, is powerful. It can quickly detect and encode the features needed to permit the type of open-ended pictorial generalization that has been well explored in this species and can also acquire large amounts of item-specific features for the purposes of instance identification.

Overall, the current results provide new insights into the sequential organization, deployment, and joint function of the cognitive mechanisms of categorization and memorization by pigeons. There are good comparative reasons to believe that these same joint interactions of categorization and memorization are critical and central to vertebrate cognition and intelligence. Examining their joint interaction in controlling behavior in a comparative context is an important future direction for research.

One key question future research should examine is the relative degree of control by these factors across different modalities and domains in other species. The current work documents a type of flexibility involving the joint determination of performance by the utility of different features, and possibly mechanisms, in pigeons. In contrast, the advanced intelligence of humans partially originates from our effective capacity to form explicit rules and concepts and rely less on rote memorization and implicit exemplar learning (Ashby, Alfonso-Reese, Turken, \& Waldron, 1998). The latter appears to be a relatively recent evolutionary development, however, as it appears that our ancestors made the same basic hominid stone toolkit, by rote with little variation, for a large portion of our past. Even now, different groups of humans seem to vary in their relative reliance and capacity to employ memorized details (Mottron, Dawson, \& Soulières, 2009; Vicari, Bellucci, \& Carlesimo, 2005). Non-human primates seem to occupy an interesting intermediate ground, being able to engage in some rule-based behavior, but not to the degree of humans (Zakrzewski, Church, \& Smith, 2018). Relatedly perhaps, some songbird species are closed-ended song learners that replicate the same song once memorized, while others continuously create new plastic versions of their songs over time (Brenowitz \& Beecher, 2005). The degree to which various animals 
can flexibly deploy these components of their cognitive toolbox is likely an important part of their natural history (Sol, Timmermans, \& Lefebvre, 2002).

Supplementary Information The online version contains supplementary material available at https://doi.org/10.3758/s13423-020-01842-4.

Open Practices Statement This experiment was not preregistered. The materials and data are available upon request from the corresponding author at Robert.Cook@tufts.edu.

\section{References}

Ashby, F. G. (Ed.) (1992). Multidimensional models of perception and cognition. Hillsdale, NJ: Lawrence Erlbaum Associates, Inc.

Ashby, F. G., Alfonso-Reese, L. A., Turken, A. U., \& Waldron, E. M. (1998). A neuropsychological theory of multiple systems in category learning. Psychological Review, 105(3), 442-481. https://doi.org/ 10.1037/0033-295X.105.3.442

Brenowitz, E. A., \& Beecher, M. D. (2005). Song learning in birds: diversity and plasticity, opportunities and challenges. Trends in Neurosciences, 28(3), 127-132. https://doi.org/10.1016/j.tins.2005. 01.004

Cook, R. G., Brown, M. F., \& Riley, D. A. (1985). Flexible memory processing by rats: Use of prospective and retrospective information in the radial maze. Journal of Experimental Psychology: Animal Behavior Processes, 11(3), 453-469. https://doi.org/10.1037/00977403.11.3.453

Cook, R. G., Levison, D. G., Gillett, S. R., \& Blaisdell, A. P. (2005). Capacity and limits of associative memory in pigeons. Psychonomic Bulletin \& Review, 12(2), 350-358. https://doi.org/10.3758/ BF03196384

Cook, R. G., \& Rosen, H. A. (2010). Temporal control of internal states in pigeons. Psychonomic Bulletin \& Review, 17, 915-922. https://doi. org/10.3758/PBR.17.6.915

Cook, R. G., \& Smith, J. D. (2006). Stages of abstraction and exemplar memorization in pigeon category learning. Psychological Science, 17(12), 1059-1067. https://doi.org/10.1111/j.1467-9280.2006. 01833.x

Daniel, T. A., Cook, R. G., \& Katz, J. S. (2015). Temporal dynamics of task switching and abstract-concept learning in pigeons. Frontiers in psychology, 6. https://doi.org/10.3389/fpsyg.2015.01334

Fagot, J., \& Cook, R. G. (2006). Evidence for large long-term memory capacities in baboons and pigeons and its implication for learning and the evolution of cognition. Proceedings of the National Academy of Science, 103, 17564-17567. https://doi.org/10.1073/ pnas.0605184103

George, D. N., \& Pearce, J. M. (2012). A configural theory of attention and associative learning. Learning \& Behavior, 40(3), 241-254. https://doi.org/10.3758/s13420-012-0078-2

Gibson, B. M., \& Wasserman, E. A. (2004). Time-course of control by specific stimulus features and relational cues during same-different discrimination training. Learning \& Behavior, 32, 183-189. https:// doi.org/10.3758/BF03196019
Herrnstein, R. J., \& Loveland, D. H. (1964). Complex visual concept in the pigeon. Science, 146(3643), 549-551. https://doi.org/10.1126/ science.146.3643.549

Katz, J. S., \& Wright, A. A. (2006). Same/different abstract-concept learning by pigeons. Journal of Experimental Psychology: Animal Behavior Processes, 32(1), 80-86. https://doi.org/10.1037/00977403.32.1.80

Minda, J. P., \& Smith, J. D. (2000). Prototypes in category learning. The effects of category size, category structure, and stimulus complexity. Journal of Experimental Psychology: Learning, Memory, and Cognition, 27, 775-779. https://doi.org/10.1037/0278-7393.27.3. 775

Mottron, L., Dawson, M., \& Soulières, I. (2009). Enhanced perception in savant syndrome: patterns, structure and creativity. Philosophical Transactions of the Royal Society B: Biological Sciences, 364(1522), 1385-1391. https://doi.org/10.1098/rstb.2008.0333

Nosofsky, R. M. (1987). Attention and learning processes in the identification and categorization of integral stimuli. Journal of Experimental Psychology: Learning, Memory, and Cognition, 13, 87-108. https://doi.org/10.1037/0278-7393.13.1.87

Qadri, M. A. J., Ashby, F. G., Smith, J. D., \& Cook, R. G. (2019). Testing analogical rule transfer in pigeons (Columba livia). Cognition, 183, 256-268. https://doi.org/10.1016/j.cognition.2018.11.011

Rayburn-Reeves, R. M., \& Cook, R. G. (2016). The organization of behavior over time: Insights from mid-session reversal. Comparative Cognition \& Behavior Reviews, 11, 103-125. https:// doi.org/10.3819/CCBR.2016.110006

Rayburn-Reeves, R. M., Qadri, M. A. J., Brooks, D. I., Keller, A. M., \& Cook, R. G. (2017). Dynamic cue use in pigeon mid-session reversal. Behavioural Processes, 137, 53-63. https://doi.org/10.1016/j. beproc.2016.09.002

Smith, J. D., Ashby, F. G., Berg, M. E., Murphy, M. S., Spiering, B., Cook, R. G., \& Grace, R. C. (2011). Pigeons' categorization may be exclusively nonanalytic. Psychonomic Bulletin \& Review, 18(2), 414-421. https://doi.org/10.3758/s13423-010-0047-8

Smith, J. D., \& Minda, J. P. (1998). Prototypes in the mist: The early epochs of category learning. Journal of Experimental Psychology: Learning, Memory, and Cognition, 24, 1411-1436. https://doi.org/ 10.1037/0278-7393.24.6.1411

Sol, D., Timmermans, S., \& Lefebvre, L. (2002). Behavioural flexibility and invasion success in birds. Animal Behaviour, 63(3), 495-502. https://doi.org/10.1006/anbe.2001.1953

Vicari, S., Bellucci, S., \& Carlesimo, G. A. (2005). Visual and spatial long-term memory: differential pattern of impairments in Williams and Down syndromes. Developmental Medicine and Child Neurology, 47(5), 305-311. https://doi.org/10.1017/ S0012162205000599

Wasserman, E. A., Kiedinger, R. E., \& Bhatt, R. S. (1988). Conceptual behavior in pigeons: Categories, subcategories, and pseudocategories. Journal of Experimental Psychology: Animal Behavior Processes, 14(3), 235-246. https://doi.org/10.1037// 0097-7403.14.3.235

Zakrzewski, A. C., Church, B. A., \& Smith, J. D. (2018). The transfer of category knowledge by macaques (Macaca mulatta) and humans (Homo sapiens). Journal of Comparative Psychology, 132(1), 5874. https://doi.org/10.1037/com0000095

Publisher's note Springer Nature remains neutral with regard to jurisdictional claims in published maps and institutional affiliations. 